\title{
Understanding Children's Attraction Toward Digital Games and Preventing Their Gaming Addiction
}

\author{
Guang-Lea Lee \\ Old Dominion University, Virginia, USA \\ Hani Morgan \\ University of Southern Mississippi, Mississippi, USA
}

\begin{abstract}
The paper attempts to bring out concerns pertaining to the rise in Korean children's over-usage of digital games, which may be harmful in many ways and is a result of increased academic pressure and high-speed Internet availability. This article focuses on school-age Korean children's addiction to computer games and the catastrophic effects it has on children. We distinguish between Internet addiction and computer game addiction, and discuss the characteristics of harmful computer games. We also explain how the extensive and excessive use of high-speed Internet increases addiction and how Korean children use computer games as a stress relieving mechanism. Additionally, we discuss the types of children who are most susceptible, as well as the dangerous consequences of addiction. Finally, we explore the efforts of the Korean government to treat gaming addiction through family counseling and an addiction rescue camp, and recommend practical suggestions for parents who can treat and prevent gaming addiction at home.
\end{abstract}

Keywords: digital games, computer games, gaming addiction, addiction treatment, Korea

\section{Introduction}

Korea has been a pioneer in designing, producing, promoting, and exporting innovative electronic devices. These devices have enhanced global connections and facilitated access to information and communication. Because Korea is a high-tech society, many children grow up by using and playing with hand-held digital devices, computers, and Internet websites. The Internet entertains children and provides them with valuable connections to their family, teachers, and friends.

Schools use the Internet for communication with students and teaching via online lessons. Even very young children spend time on the Internet, both at home and in school, to complete their academic work. This online presence provides students with a sense of real-world relevance, convenient ways to collaborate and communicate entertainment outlets, and opportunities to broaden their educational horizons (Borup, 2016). However, an adverse effect of this high-tech society is students' obsessive use and dependence upon electronics and high-speed connectivity.

Korea boasts both the highest percentage of households with wireless Internet connection and the highest

Guang-Lea Lee, Ph.D., professor, Department of Teaching and Learning, Old Dominion University.

Hani Morgan, Ed.D., associate professor, Department of Curriculum, Instruction, and Special Education, University of Southern Mississippi. 
speed broadband networks in the world. Both have contributed to many Korean children's addiction to the Internet and gaming, and to their craving for electronic games. As Robinson (2015) stated that their Internet addiction crisis was getting worse, as some teens spend up to 88 hours a week gaming online. Addiction to computer gaming has led to consequences, such as fights, and in some cases, even death. Several young men have collapsed and died while playing computer games in Internet cafes (Elise, 2015; Griffiths, 2015). These young men had not slept properly and had eaten very little during their gaming sessions, which lasted more than 48 hours. The causes of deaths were found to be heart failure due to exhaustion.

\section{Korea's National Issue of Gaming Addiction}

Gaming addiction in Korea has become a national issue in recent years, in part because several gamers have died from exhaustion after playing for days on end with no breaks. After 10 cardiopulmonary-related deaths in Internet cafes and a murder involving computer game users, Korea now considers game addiction one of its most serious mental health issues. The computer gaming addiction rate for elementary school students in Korea is $14 \%$, twice the rate of that for adults. The average addiction rates for middle and high school students are $12 \%$ and $10 \%$, respectively (National Information Society Agency [NIA], 2012).

\section{Internet Addiction vs. Gaming Addiction}

Internet addiction is a broad term and involves a variety of activities on the Internet that lead to psychological dependence, while gaming addiction is one type of Internet addiction. Internet addicts over-use the Internet for various purposes, including research, learning, social networking, gambling, shopping, and computer gaming. Four commonly used terms referring to maladaptive patterns of Internet use are: (a) Internet addiction (Telef, 2016; Weinstein \& Lejoyeux, 2015); (b) Internet addiction disorder (Bell, Choudhury, Guyot, \& Meier, 2016; Li, O’Brien, Snyder, \& Howard, 2015); (c) Internet pathological use (Hinvest \& Brosnan, 2012); and (d) Internet dependency (Chou, Condron, \& Belland, 2005; Sun, Rubin, \& Haridakis, 2008). Toker and Baturay (2016) found that newer computer games are more likely to addict children, since they contain improved and more appealing sounds and visual effects, when compared to older video arcade games. Children experience such attraction while playing on various gaming platforms and hand-held devices. Therefore, computer game addiction falls on the compulsive-impulsive spectrum disorder and involves excessive computer usage both online and off (Dell'Osso et al., 2006). Computer game addiction consists of four unhealthy characteristics: (a) excessive use; (b) withdrawal; (c) tolerance; and (d) negative repercussions (Beard \& Wolf, 2001; Block, 2008; Brack et al., 2013).

Gamers who become "hooked" experience difficulty with self-control, and play every day for extended periods of time (over four hours), get restless or irritable if they cannot play, and sacrifice other social activities to play games (Center for Internet Addiction Recovery, 2017). Similar to the behavior of other addicts, children addicted to computer games isolate themselves, reject the real world, and turn to computer games as a way to escape the pressures of the real world.

\section{Children Vulnerable for Gaming Addiction}

Although both children and adults can become addicted, young children suffer the most in general. This is due to the fact that their addiction interferes with their ability to function normally and to participate in routine daily activities, such as getting out of bed to go to school. This can be detrimental to their normal development. Astonishingly, $30 \%$ Korean children under the age of 18 years or about 2.4 million people are at risk of Internet 
addiction and require psychological support. Educators in South Korea acknowledge the need for counseling for children who show signs of gaming addiction (NIA, 2012).

Seo and Lim (2010) warned that even pre-schoolers and elementary students are at risk and found that children suffering from depression, anxiety, or paranoia are at higher risk of becoming addicted. When using smartphones or other digital devices, such children become excited, in some cases, forgetting to eat meals but they are distraught when these devices are taken away from them. Children who are from unstable families, in abusive relationships, or have introverted personalities are more inclined to become deeply involved in fantasy games, and therefore, are more prone to computer addiction. They often do not have a loving family, so they are detached from others and lack emotional bonding. Their parents are often unsupportive and tend to use unreasonable and harsh parenting practices (Ann, 2000; Park, 2001).

A survey conducted by the NIA (2012) reported that under-privileged students without concerned and loving families were more likely to be addicted to gaming. Typically, these children have insufficient communication with their parents and lack a proper role model at home. Additionally, children with computer game addiction commonly suffer from emotional problems, such as depression, stress, and anxiety-related disorders (Lim et al., 2015). They tend to be violent, aggressive, impulsive, anxious, and have low self-confidence (Song $\&$ Sim, 2003). These children are drawn to computer games, since they often use the fantasy world of the computer games to psychologically escape unpleasant feelings or stressful situations (Young \& Rodgers, 1988). In their cyber place, they feel safe and cocooned in their fantasy world, often playing games for hours.

\section{Games as a Stress-Relieving Mechanism for Students in Korea}

Korean children are under constant pressure to succeed academically and most of their time after school is spent on tasks related to academics. They study rigorously because of the strong emphasis placed on earning high scores on examinations and ranking higher than their peers. They often endure excessive stress in this highly competitive academic atmosphere. When they fail to meet their parents' academic expectations, students often become depressed. Many depressed students become rebellious in response to their parents' expectations and look for ways to relieve their stress. Korean parents and teachers tend to permit children to play games, because they understand and sympathize with students who are stressed from their studies. Their support stems from the notion that computer gaming is a way to alleviate the stress that comes from spending three to four additional hours daily at an after school tutoring institute, and then, arriving at home to do even more schoolwork. Reinecke (2009, p. 126) reported that playing computer games can actually help individuals who are dealing with stress and strain in their daily lives. While gaming, the players can "escape negative thoughts about themselves."

Since Korea has few parks or fields where children can play outdoors, young people often relieve their intense academic stress by playing computer games. Excessive homework, tight study schedules, and extra classes after school can cause some children to retreat into a cyber-world where they feel free, in control, and secure. They may desire to play games in between studies or frequently look for "personal computer (PC) rooms" in order to decrease their stress and enjoy interaction with both real and virtual peers. In "PC rooms," also called "Internet parlors" or "Internet cafes," children play computer games with others. These places are a type of neighborhood community center, coffee shop, day care center, shelter, study hall, and game room all in one. Often after school, instead of gathering at an after-school club, students go to a PC room, one of which can be found on almost every block in Korea, to play with their friends in virtual space. Children admire successful 
computer gamers in Korea, who are considered "e-athletes." Internet gaming events, known as "e-sports" are broadcast on television the same as traditional competitive sports. The e-athletes who compete in the annual World Cyber Games (2017) were treated like sports celebrities and possess gaming prowess that children want to emulate.

\section{Government Efforts for Treating Gaming Addiction}

\section{Family Counseling}

Addicted children spend at least two hours a day online playing games and are usually unable to stop on their own. They tend to play for longer and longer periods and experience withdrawal symptoms, such as anger, when prevented from logging on. Child psychiatrists and child development experts recommend that parents seek counseling for children at risk. They advise parents to contact their local general practitioners who sometime refer children to a psychologist for counseling. Many Korean parents, however, ignore this recommendation because of their traditional cultural views regarding psychotherapy. Psychological treatment for mental health issues is considered a sign of spiritual weakness and often brings shame to the family. Koreans believe that psychological counseling is intended for patients with severe schizophrenic illness. They believe that addictive behaviors can be overcome with strong resistance to temptation by using will power. In an effort to combat this prejudice toward counseling and provide needed treatment, the government has initiated counseling centers throughout Korea.

The Ministry of Public Administration and Security created the NIA and launched 140 Internet addiction counseling centers across Korea, including a mobile counseling van in each city that targets PC parlors. The counseling centers have trained over 2,300 professional counselors solely for the treatment of this problem. They promote the proper use of the Internet and treat those unable to control their use of it. They offer free counseling services for addicted children and teens, conduct Internet addiction prevention campaigns, and administer surveys on Internet addiction. Also, the Ministry of Gender Equity and Family has addressed Internet addiction issues by launching the Korea Youth Counseling Institute in 2017 to help out-of-control youth who are unable to stop playing Internet games and media. These governmental efforts contributed to Korea being a leader in treating Internet and gaming addiction (Young, 2015) to help families with problems arising from the Internet.

\section{Addiction Rescue Camp}

This tuition-free, government-funded gaming and Internet addiction camp is the first of its kind in the world. The camp uses the acronym "RESCUE," which represents the goals of treatment: re-experience new activities in the camp (R); feel excitement (E); socialize with peers, mentors, and instructors (S); face challenge for change (C); unite with family members (U); and escape computer addiction (E). The Internet RESCUE Camp is located in a forested area about an hour south of the capital city of Seoul. It offers room and board, assesses participants, assigns mentors, and works in collaboration with medical professionals. While it is too early to know its long-term success rate, the camp receives four to five applications for each spot available. The schedule at the camp includes exercise as well as group leisure activities, such as horseback riding. The purposes of these activities are to build emotional connections to real life leisure, to weaken the connection to virtual games, and to provide victims with the experience of a lifestyle other than that on the Internet (Cornish, 2016). 


\section{Recommendations for Parents to Prevent Gaming Addiction}

\section{Supervise and be Involved at Home}

Children need adult attention, supervision, and guidance when using computers. Therefore, parents should monitor their children. Before children develop an excessive interest in computer games, parents need to find extra-curricular learning and sport activities for them, in order to divert their attention away from computers. Families can plan leisure activities and encourage children to participate in alternative forms of entertainment. As role models, parents should demonstrate the importance of participating in a variety of experiences and maintaining healthy social relationships.

Parents can show interest in the computer games their children play by participating, discussing, and interacting with them during the game. They can even make computer games a part of family fun. For example, parents can discuss the content and rules of the game. Parents and children should agree on specific time limits for computer use. The Korean Education Development Institute recommends that young children aged 5-12 use the Internet only once a day for no more than 30 minutes and asks parents to designate one day a week "no Internet day" (Chun, 2008). The Korean government enforces a "shut-down law" which prohibits children under the age of 16 from playing games between midnight and six o'clock in the morning (Young, 2015). Parents would benefit from having such a rule in their own home as well.

To decrease the risk of their children developing of a computer addiction, parents can install games only on a computer located in a high-traffic area of the house. With others looking over their shoulder, it is believed children will be less likely to over-use the computer. Parents can also control login and password information to restrict children's access to online games that they visit compulsively. When creating a password, parents can refrain from sharing it with their children, so that children are only able to play games under their supervision and for a set amount of time.

\section{Promote Socialization and Educational Games}

Parents need to help children succeed academically and socially, assist them with their schoolwork, and encourage them to spend time with their real-life friends before playing games. When children have no friends to play with, they seek other ways to connect with others, such as through computer games. Parents and teachers need to find out if their children are isolated or bullied at school and to end these behaviors if they are occurring. They need to provide games that benefit children educationally and emotionally. Many computer games feature violent themes. Parents need to teach their children how to differentiate between beneficial and harmful video games and protect them from excessive exposure to violent games by teaching them about violence and addiction. They should be present when their children are purchasing games and ensure that children select games that contribute to desired results. Furthermore, they need to be familiar with the content of games and stand ready to provide children with alternative games, such constructing a city or caring for pets. They need to provide young children with games that promote learning and encourage positive, prosocial behavior by rewarding players who cooperate or share.

\section{Be Knowledgeable and Aware}

In addition, parents need to know which PC parlors their children visit and keep track of the games children like to play and the number of hours they play away from home. Parents need to be aware of the names of violent games. They can use the rating system developed by the Entertainment Software Ratings Board 
(ESRB) to check the degree of violence and age appropriateness of the game their child is playing. Finally, parents need to be aware of the signs of addicted gamers, so they can get professional help for their children as early as possible. Like drug addicts, gaming addicts show clear warning signs. Common early warning signs that children may exhibit include preoccupation with games, lying about the games, losing interest in other activities, withdrawing from family and friends, and using games to escape the real world. When a child demonstrates any of these signs, parents need to pay close attention and seek professional help.

\section{Conclusions}

Unlike the help available for traditional addictions, such as alcohol and gambling, few support groups and treatment centers exist for computer game addiction even in many of the world's high-tech countries. However, Korea is a leader in addressing the growing risks associated with its advanced cyber culture. Korea has made significant gains in defining and researching this problem after recognizing that many of its young people suffer from a major computer addiction issue. Korean treatment programs focus on preventive measures and strategies for schools and homes. For example, in order to help educators and parents recognize addiction, researchers at the Korea Internet Addiction Center (2017) had developed diagnostic criteria to help parents of preschool and primary school age children to observe their children's behavior and measure their level of Internet game addiction.

To protect children from violent content and addiction, government policy-makers and game developers need to impose age restrictions on games designed for mature users. Disclosure of risk factors and game themes should be noted on game packaging in order to help parents both protect their children and monitor violent content. Internet game developers and service providers should follow the guidelines set by the Ministry of Culture, Sports, and Tourism (2012) to prevent children's Internet game addiction. The National Assembly Research Service (2011) had established curfews and set time limits of between midnight and six o'clock in the morning that forbid children younger than 16 years of age from playing in Internet cafes. Parents should apply similar precautions at home. Such efforts will reduce Internet gaming addiction in Korea. These efforts will also prevent other countries throughout the world that have not yet developed a serious problem from experiencing the devastating consequences of computer game addiction.

\section{References}

Ann, S. (2000). Study about addictive use of Internet: Focusing on middle school students in Seoul (Master's thesis, Yonsei University, Seoul, Korea).

Beard, K. W., \& Wolf, E. M. (2001). Modification in the proposed diagnostic criteria for Internet addiction. Cyber Psychology and Behavior, 4(3), 377-383.

Bell, R., Choudhury, D., Guyot, W. M., \& Meier, R. J. (2016). Impulse control and Internet addiction disorder among business professionals. Journal of Organizational Culture, Communications, and Conflict, 20(1), 1-19.

Block, J. J. (2008). Issues for DSM-V: Internet addiction. American Journal of Psychiatry, 165(3), 306-307.

Borup J. (2016). Teacher perceptions of learner-learner engagement at a cyber high school. International Review of Research in Open \& Distance Learning, 17(3), 231-250.

Brack, G., Lassiter, P. S., Kitzinger, R., Hill, M., McMahon, H. G., \& Fall, K. A. (2013). Individual psychology on the virtual frontier: Massive multi-player online role-playing gaming. The Journal of Individual Psychology, 69(1), 24-40.

Center for Internet Addiction Recovery. (2017). Internet gaming disorder. Retrieved October 10, 2017, from http://netaddiction. com/online-gaming/

Chou, C., Condron, L., \& Belland, J. C. (2005). A review of the research on internet addiction. Educational Psychology Review, 17(4), 363-388.

Chun, L. S. (2008). Problem-solving strategies for dysfunction of information oriented society: Internet addiction. Education Policy Forum, 175, 8-12. 
Cornish, D. (2016, April). Korea's Internet addicts. Retrieved October 15, 2017, from http://www.sbs.com.au/news/dateline/ story/koreas-internet-addicts

Dell'Osso, B., Altamura, A. C., Allen, A., Marazziti, D., \& Hollander, E. (2006). Epidemiologic and clinical updates on impulse control disorders: A critical review. European Archives of Psychiatry and Clinical Neuroscience, 256(8), 464-475.

Elise, A. (2015, January). Gamer dies in Internet cafe after three day gaming binge, hardly anyone notices. Retrieved October 15, 2017, from http://www.ibtimes.com/gamer-dies-internet-cafe-after-three-day-gaming-binge-hardly-anyone-notices-1787936

Griffiths, M. (2015, January). Gaming to death: What turns a hobby into a health hazard? Retrieved October 15, 2017, from http://www.cnn.com/2015/01/21/opinion/gaming-addiction-risks/

Hinvest, N., \& Brosnan, M. (2012). Identifying vulnerability markers for pathological internet use and pathological video game playing within an educational context. Journal of Educational Computing Research, 46(4), 357-376.

Korea Internet Addiction Center. (2017). Preschool and primary students' game addiction scale. Retrieved October 20, 2017, from http://www.iapc.or.kr/dia/survey/addDiaSurveyNew.do?dia_type_cd=GABO

Korea Youth Counseling Institute. (2017). Internet addiction. Retrieved October 20, 2017, from https://www.kyci.or.kr/userSite/ sub02_3.asp

Li, W., O’Brien, J. E., Snyder S. M., \& Howard, M. O. (2015). Characteristics of Internet addiction/pathological Internet use in U.S. university students: A qualitative-method investigation. PLOS ONE, 10(2), 1-19.

Lim, J. A., Gwak, A. R., Park, S. M., Kwon, J. G., Lee, J. Y., Jung, H. Y., \& Choi, J. S. (2015). Are adolescents with internet addiction prone to aggressive behavior? The mediating effect of clinical comorbidities on the predictability of aggression in adolescents with Internet addiction. Cyberpsychology, Behavior, and Social Networking, 18(5), 260-267.

Ministry of Culture, Sports, and Tourism. (2012). Preventative measures of game addiction. Seoul, Korea.

National Assembly Research Service. (2011). Implementation of game shut-down system and directions for future policies. Seoul, Korea: National Assembly.

National Information Society Agency (NIA). (2012). Internet addiction survey 2011 summary report (NIA IV-RER-11052). Seoul, Korea: National Information Society Agency.

Park, J. E. (2001). Teen's addictive use of Internet and factors of addiction (Master's thesis, Sook Myung Women's University, Seoul, Korea).

Reinecke, L. (2009). Games and recovery: The use of video and computer games to recuperate from stress and strain. Journal of Media Psychology: Theories, Methods, and Applications, 21(3), 126-142.

Robinson, M. (2015, March). Korea's Internet addiction crisis is getting worse, as teens spend up to 88 hours a week gaming. Retrieved October 20, 2017, from http://www.businessinsider.com/south-korea-online-gaming-addiction-rehab-centers-2015 -3 .

Seo, M. Y., \& Lim, E. M. (2010). Infants' and low-grade elementary students' Internet game addiction tendency and the relationship between game addiction tendency and personality characteristic. The Journal of Child Education, 19(4), 163-175.

Song, S. J., \& Sim, H. O. (2003). Computer game immersion and children`s psychosocial/behavioral characteristics. The Journal of Korea Child Study, 24(5), 27-41.

Sun, S., Rubin, A. M., \& Haridakis, P. M. (2008). The role of motivation and media involvement in explaining Internet dependency. Journal of Broadcasting \& Electronic Media, 52(3), 408-431.

Telef, B. B. (2016). Investigating the relationship among Internet addiction, positive and negative affects, and life satisfaction in Turkish adolescents. International Journal of Progressive Education, 12(1), 128-135.

Toker, S., \& Baturay, M. H. (2016). Antecedents and consequences of game addiction. Computers in Human Behavior, 55, 668-679.

Weinstein, A., \& Lejoyeux, M. (2015). New developments on the neurobiological and pharmaco-genetic mechanisms underlying Internet and videogame addiction. American Journal on Addictions, 24(2), 117-125.

World Cyber Games. (2017). About the world cyber games of the past. Retrieved October 20, 2017, from http://worldcybergames. $\mathrm{com} /$

Young, K. (2015). The evolution of Internet addiction disorder. In C. Montag \& M. Reuter (Eds.), Internet addiction: Neuroscientific approaches and therapeutical interventions (pp. 3-20). New York, N.Y.: Springer.

Young, K. S., \& Rodgers, R. C. (1988). The relationship between depression and internet addiction. Cyber Psychology \& Behavior, 1(1), 25-28. 